\title{
Evaluación de la relación de los signos y síntomas durante el período de erupción de los dientes primarios.
}

\author{
Rangel Bastos de Holanda Teixeira, ${ }^{1}$ \\ Lorena de Alencar Gonçalves Ferreira do Amaral, ${ }^{1}$ \\ Dayse Andrade Romão, ${ }^{2}$ \\ Mariana Alencar Nemezio ${ }^{3}$
}

\section{Resumen}

Introducción: La erupción dentaria es un proceso fisiológico que consiste en la migración del diente de su posición intraósea en la mandíbula o el maxilar hacia la cavidad bucal. Se asocia a algunos signos y síntomas como irritabilidad, irritación gingival, aumento de la salivación, sueño agitado, diarrea, pérdida de apetito y fiebre. Objetivo: Evaluar si las madres relatan la presencia de signos y síntomas durante la erupción de los dientes primarios y si las actitudes tomadas por los responsables en relación a la sintomatología interfieren en la condición del niño. Materiales y Métodos: Se recogieron datos de niños $(n=50)$ entre 5 y 24 meses de edad. Los signos y síntomas observados por las madres durante la erupción de los dientes primarios y las actitudes tomadas por los responsables en relación a la sintomatología, fueron evaluados por medio de cuestionario. Resultados: La prueba de Chi-cuadrado $(0,010)$ reveló haber asociación de las signos y síntomas con la emergencia de los dientes primarios. Para actitudes tomadas por los responsables en relación a la sintomatología, la prueba del Chicuadrado fue de 0,009 mostrando una influencia significativa en la muestra. Conclusión: Se puede afirmar que las madres relatan la aparición de signos y síntomas durante la emergencia de los dientes primarios y actitudes tomadas por los responsables en relación a la sintomatología contribuyen a mejorar la condición de los niños.

Palabras clave: Erupción Dentaria; signos y síntomas; diente primario.

\footnotetext{
${ }^{1}$ Graduando de Odontologia - Centro Universitário Tiradentes - UNIT, Alagoas, Brasil.

${ }^{2}$ Profesora - Doctora - Centro Universitário Tiradentes - UNIT, Alagoas, Brasil.

${ }^{3}$ Profesora Orientadora - Doctora - Centro Universitário Tiradentes - UNIT, Alagoas, Brasil.
} 
Artigo original

\section{Avaliação da relação dos sinais e sintomas durante o período de erupção dos dentes decíduos.}

\section{Resumo}

Introdução: A erupção dentária é um processo fisiológico, consiste na migração do dente da sua posição intraóssea na mandíbula/maxila para a cavidade bucal. Está associada a alguns sinais e sintomas como irritabilidade, irritação gengival, aumento da salivação, sono agitado, diarreia, perda de apetite e febre. Objetivo: Avaliar se as mães relatam a ocorrência dos sinais e sintomas durante a erupção dentes decíduos e se atitudes tomadas pelos responsáveis em relação à sintomatologia interferem na condição da criança. Material e Método: Foram coletados dados de crianças $(n=50)$ com idade de 5 a 24 meses. Os sinais e sintomas observados pelas mães durante a erupção dos dentes decíduos e atitudes tomadas pelos responsáveis em relação à sintomatologia, foram avaliados por meio de questionário. Resultados: O teste do Qui-quadrado $(0,010)$ revelou haver associação dos sinais e sintomas com o irrompimento dos dentes decíduos. Para atitudes tomadas pelos responsáveis em relação à sintomatologia, o teste do Qui-quadrado foi de 0,009 mostrando uma influência significativa na amostra. Conclusão: Pode-se concluir que as mães relatam a ocorrência de sinais e sintomas durante o irrompimento dos dentes decíduos e atitudes tomadas pelos responsáveis em relação à sintomatologia contribuem para melhora na condição das crianças.

Palavras chaves: Erupção Dentária; sinais e sintomas; dente decíduo.

Original article

\section{Evaluation of the relationship of signs and symptoms during the period of eruption of deciduous teeth.}

\begin{abstract}
Introduction: Dental eruption is a physiological process, consisting of the migration of the tooth from its intraosseous position in jaw bones to buccal cavity. In infants it is associated with signs and symptoms such as irritability, gingival irritation, increased salivation, restless sleep, diarrhea, loss of appetite and fever. Objective: To assess mothers reports the occurrence of signs and symptoms during the
\end{abstract}

eruption of deciduous teeth and whether the attitudes taken improve the child's condition. Material and Method: Data was collected from children $(n=50)$ aged 5 to 24 months. The signs and symptoms observed by the mothers during the eruption of deciduous teeth and attitudes taken by those responsible for the child were evaluated by means of a questionnaire. Results: The chi-square test (0.010) revealed an association of signs and symptoms with eruption of deciduous teeth. For the attitudes 
taken by the guardians regarding the symptomatology, the Chi-square test showed significantinfluencein the sample (0.009). Conclusion: It can be concluded that the mothers report the occurrence of signs and symptoms during the eruption of deciduous teeth and the attitudes

\section{Introducción}

La erupción dentaria es un proceso fisiológico natural que generalmente ocurre sin problemas. Consiste en la migración del diente de su posición intraósea en la mandíbula y en la maxila para la emergencia en la cavidad bucal. Diferentes tejidos y mecanismos fisiológicos están involucrados. ${ }^{1,2}$

La erupción de los dientes primarios se inicia entre los 4 a 10 meses después del nacimiento, quedando completa con 20 dientes, en general en el $30^{\circ}$ mes. 5 El proceso eruptivo está bajo fuerte control genético con menor influencia de factores ambientales. ${ }^{6}$ Ha sido asociado a algunos signos y síntomas que incluyen irritabilidad, irritación gingival, aumento de la salivación, sueño agitado, diarrea, pérdida de apetito y fiebre..$^{7-12}$

Estudios demuestran que el malestar durante la emergencia de los dientes primarios puede coincidir con la disminución de la inmunidad humoral materna y el establecimiento de inmunidad humoral del niño. ${ }^{3,13,14}$ Se observa que estas molestias disminuyen con la edad, que pueden estar asociadas a la inmunidad o no. ${ }^{15}$ taken by those responsible in relation to the symptomatology contribute to an improvement in the condition of the children

Key words: Tooth eruption; signs and symptoms; primary tooth.

El surgimiento de los signos y síntomas durante la erupción dentaria sigue siendo controvertido., ${ }^{216-19}$ No hay evidencias para indicar si estos hallazgos clínicos ocurren debido la erupción de los dientes primarios. ${ }^{10,20-24}$ Cuando están presentes pueden ser usados métodos para la disminución del descontento del niño durante la erupción de los dientes primarios. Geles que contienen anestésicos, analgésicos y mordedor de silicona pueden ser utilizados por los responsables. ${ }^{13}$ Sin embargo, los padres no reciben orientación médica ni odontológica para el alivio de los síntomas. ${ }^{25}$

Deesta forma, el objetivo deesteestudiofue evaluar si las madres relatan la ocurrencia de los signos y síntomas durante la erupción de los dientes primarios y si las actitudes tomadas por los responsables en relación a la sintomatología interfieren en la condición del niño.

\section{Materiales y métodos}

Este trabajo fue aprobado por el Comité de Ética en Pesquisa del Centro Universitario Tiradentes, Maceió - AL (CAAE: 75624017.2.0000.5641).

Fue realizado un estudio observacional con duración de 12 meses, por medio de 
cuestionario. Cincuenta madres con hijos de 5 a 24 meses de edad en la ciudad de Maceió - AL, Brasil participaron en la investigación. Todas las madres recibieron el cuestionario y el consentimiento libre e informado explicando la naturaleza de la investigación, su importancia y la necesidad de obtener respuesta correcta.

Los participantes elegibles incluyeron niños de 5 a 24 meses de edad sin antecedentes de enfermedad crónica o disturbios que podrían influir en los resultados del estudio. Antes de la recolección de los datos se realizó un estudio piloto con 10 niños entre 5 a 24 meses de edad, para estandarización de la metodología. Después del estudio piloto se inició la recolección de los datos, por un período de 8 meses.

El cuestionario utilizado en el estudio contenía preguntas sobre signos y síntomas relacionados a la erupción dentaria y sobre las actitudes tomadas por los responsables en relación a la sintomatología (Figura 1). Dos estudiantes de Odontología calificados realizaron el examen de la cavidad bucal para determinar si la erupción dentaria estaba ocurriendo. El examen clínico fue realizado en la casa de los niños usando una linterna para proporcionar una fuente de luz estandarizada asociada a la palpación (dedo índice en la cresta alveolar). La recolección de datos se inició con la erupción de al menos uno de los incisivos. Las madres fueron interrogadas acerca de los métodos de alivio de los signos y síntomas de la erupción dentaria, bien como la ocurrencia de irritabilidad, irritación gingival, aumento da salivación, sueño agitado, diarrea, pérdida de apetito, evaluados por medio de cuestionario.

\section{CUESTIONARIO}

NOMBRE DE LA MADRE:

NOMBRE Y EDAD DEL NIN̈O:

DIRECCIÓN:

TELĖFONO:

OBSERVACIONES:

1. ¿Cuales los métodos que tu has utilizado para el alivio de los sintomas de la erupción dentaria?

2. ¿Cuales fueron las señales y sintomas observados durante la erupción de los dientes primarios?

\begin{tabular}{|l|l|l|l|}
\hline Fiebre & Gripe & Rinorrea & Sialorrea \\
\hline Tos & Irritabilidad & Edema Gingival & Diarrea \\
\hline Vómito & Succíon Digital & Inapitencia & Rubor Facial \\
\hline Distúrbio Del Sueño & Conjuntivitis & Úlceras Bucales & Convulcio \\
\hline Sin Sintomatología & & & \\
\hline
\end{tabular}

Figura 1: Cuestionario utilizado para el análisis de los signos y síntomas durante la erupción de los dientes primarios y métodos de alivio utilizados por los responsables en relación a la sintomatología. 
Los datos obtenidos fueron digitados y las variables dependientes de este estudio fueron: síntomas y actitudes tomadas por los responsables en relación a la sintomatología. Para el análisis estadístico de la prueba Chi-cuadrado fue utilizado el programa SPSS para Windows versión 20.0, en todos los análisis se consideró el nivel de significancia del 5\%. Estos mismos datos, también fueron analizados de forma descriptiva (análisis de porcentaje).

\section{Resultados}

\section{Análisis estadístico:}

La prueba del Chi-cuadrado $(0,010)$ resultó tener asociación con los signos y síntomas y la emergencia de los dientes primarios, mostrando desviaciones significativas, habiendo asociación entre los grupos (variables independientes).

Para las actitudes tomadas por los responsables en relación a la sintomatología a la prueba del Chi-cuadrado fue de 0,009, mostrando influencia significativa en la muestra, aceptando la hipótesis de que el método de alivio interfiere en la condición del niño.

\section{Análisis descriptivo:}

Fueron realizados 50 cuestionarios, siendo todos respondidos integralmente $(100 \%)$, por las madres.

Tabla 1. Signos y síntomas relatados por las madres durante el período de erupción de los dientes primarios.

\begin{tabular}{|l|c|c|}
\hline Síntomas y signos & $\begin{array}{c}\text { Número de Incidencias de la } \\
\text { muestra }\end{array}$ & $\begin{array}{c}\text { Porcentaje de prevalencia en la } \\
\text { muestra }\end{array}$ \\
\hline Morder Objetos & 34 & $68 \%$ \\
\hline Sialorrea & 32 & $64 \%$ \\
\hline Edema Gingival & 30 & $60 \%$ \\
\hline Irritabilidad & 29 & $58 \%$ \\
\hline Diarrea & 28 & $56 \%$ \\
\hline Fiebre & 21 & $42 \%$ \\
\hline Vómito & 17 & $34 \%$ \\
\hline Disturbio del Sueño & 17 & $34 \%$ \\
\hline Dolor & 13 & $26 \%$ \\
\hline Gripe & 8 & $16 \%$ \\
\hline Tos & 8 & $16 \%$ \\
\hline Rinorrea & 7 & $14 \%$ \\
\hline Estreñimiento & 2 & $4 \%$ \\
\hline Úlceras Bucales & 2 & $4 \%$ \\
\hline Eritema Gingival & 1 & $2 \%$ \\
\hline
\end{tabular}


Tabla 2. Porcentajes de las actitudes tomadas por los responsables en relación a la sintomatología.

\begin{tabular}{|l|c|c|}
\hline $\begin{array}{l}\text { Actitudes tomadas por los } \\
\text { responsables en relación a la } \\
\text { sintomatología }\end{array}$ & $\begin{array}{c}\text { Número de Incidencias } \\
\text { De la muestra }\end{array}$ & $\begin{array}{c}\text { Porcentaje de prevalencia } \\
\text { De la muestra }\end{array}$ \\
\hline Automedicación & 22 & $44 \%$ \\
\hline Mordedores & 22 & $44 \%$ \\
\hline Geles Anestésicos & 14 & $28 \%$ \\
\hline Sin Métodos & 7 & $14 \%$ \\
\hline Chupete Helado & 3 & $6 \%$ \\
\hline Consulta Médica/Odontológica & 1 & $2 \%$ \\
\hline
\end{tabular}

\section{Discusión}

El surgimiento de los signos y síntomas durante la emergencia dentaria es controvertido. Algunos estudios demuestran que síntomas específicos están asociados a la erupción dentaria, 10,20-23 a pero aún no existen evidencias para indicar que los actos del mecanismo proceden de la erupción de los dientes primarios. ${ }^{15,24}$

En este estudio todas las madres relataron sintomatología durante la emergencia de los dientes primarios, estos resultados también han sido verificados por otros autores. ${ }^{12,23,26,27}$ Sin embargo, para los pediatras sólo algunos niños presentan estos hallazgos clínicos $^{28}$ difiriendo a lo encontrado en este estudio. Para los dentistas la mayoría de los niños presentan síntomas durante ese período, mostrando resultados similares a nuestra investigación. ${ }^{28}$

En el presente estudio, morder objetos, sialorrea, inflamación gingival, irritabilidad y diarrea fueron los factores más descritos por las madres, resultados similares fueron observados por otros investigadores. ${ }^{26}$ Estos resultados difieren de otros estudios en el cual la fiebre $^{29}$ y edema gingival fueron los más reportados. ${ }^{30}$

El signo más relatado en este estudio fue morder objetos, siendo el más observado también por otros autores. ${ }^{10,31-33}$ Esto puede ser justificado por el hecho de la incomodidad durante la erupción de los dientes primarios que puede ser aliviada por la compresión de las encías durante la mordida. ${ }^{34}$

La sialorrea fue la segunda sintomatología más reportada por las madres en la presente investigación, este resultado también fue similar en otros estudios. $10,23,31,32,35 \mathrm{El}$ aumento del flujo salival es debido a la maduración de las glándulas salivales y por la reducida capacidad de tragar del niño que coinciden con el período eruptivo de los dientes primarios. ${ }^{8}$

La inflamación gingival fue relatada por $60 \%$ de las madres, siendo también 
descrita en otro estudio. ${ }^{31}$ Este sígno puede estar relacionado a la presencia de inmunoglobulina $\mathrm{E}(\mathrm{IgE})$ en los tejidos circundantes a los dientes en erupción. ${ }^{36}$

El cuarto síntoma más citado por las madres fue irritabilidad, también siendo una alteración frecuente en otros estudios. ${ }^{23,37-39}$ Los padres relataron en otra investigación ${ }^{33}$ que el dolor en la encía puede llevar a la irritabilidad.

En este estudio, la diarrea fue relatada por $56 \%$ delas madres, siendola sintomatología menos relatada. Esta información también fue observada por otra investigación. ${ }^{40} \mathrm{Sin}$ embargo, otros estudios encontraron una prevalencia mayor. ${ }^{27,29,41,42} \mathrm{La}$ diarrea es un trastorno intestinal que puede no estar relacionado a la erupción de los dientes y si a una infección bacteriana, pudiendo ser consecuencia de la contaminación de los dedos y/o objetos llevados a la boca por los niños. ${ }^{16}$

En relación a los métodos utilizados para el alivio de la incomodidad de los signos y síntomas, se nota que los más usados por las madres eran analgésicos y mordedores, seguidos por las pomadas anestésicas. Otros estudios ${ }^{21,31,32}$ encontraron conductas similares a las relatadas por las madres en esta investigación. El uso de mordedores para promover el alivio de la sintomatología fue previamente reportado. ${ }^{43}$ Por otro lado, los analgésicos, como el paracetamol, son empleados de forma amplia por los padres con el objetivo de cesar el dolor del niño, pues promueven el bloqueo de los receptores sensoriales. ${ }^{44}$ Se observa también que los geles anestésicos tienen un efecto casi inmediato en la disminución de la incomodidad del niño, debido a su acción local sobre la región inflamada. Este efecto es debido a la lidocaína (anestésico tópico local), presente en su composición química. ${ }^{45}$

En la muestra de las actitudes tomadas por los responsables en relación a la sintomatología (Tabla 2), 14\% de las madres no hicieron uso de ningún método de alivio. Los responsables informaron que esto fue debido al hecho de los niños presenten signos clínicos leves, como la sialorrea y falta de apetito, siendo que la automedicación, uso de mordedores, geles anestésicos, no controlan tales síntomas.

En este estudio hubo limitaciones debido a la negativa en la autorización de la realización del examen de la cavidad bucal por parte de algunos de los representantes para determinar si la erupción dentaria estaba ocurriendo. Sin embargo, los factores de confusión fueron reducidos por la exclusión de esa muestra, o sea, sólo los niños en los que la erupción dental estaba ocurriendo hicieron parte de la muestra.

\section{Conclusión}

Se puede afirmar que las madres relatan la aparición de signos y síntomas durante la emergencia de los dientes primarios y que actitudes tomadas por los responsables en relación a la sintomatología contribuyen a mejorar la condición de los niños.

\section{Agradecimientos}

Al profesor Dr. Rodrigo Galo por realizar los datos estadísticos; Al PIBIC- FAPEAL por conceder la beca para realización de la investigación en 2017. 


\section{Referencias bibliográficas}

1. Massier M, Schour I. Studies in tooth development: theories of eruption. Am J Orthod. 1941; 27: 522-576.

2. Craddock H, Youngson C. Eruptive tooth movement - the current state of knowledge. British Dental Journal, 2004; 197: 385-391.

3. Baykan Z, Sahin F, Beyazova U, Ozcjakar B, Baykan A. Experience of Turkish parents about their infants' teething. Child Care Health Dev. 2004; 30: 331-336.

4. Guedes Pinto AC. Odontopediatria. São Paulo: Santos. 8 ed. 2010. 970p.

5. Folayan M, Owotabe F, Adejuyigbe E, Sen, S, Lawal B, Ndukwe K. The timing of the primary dentition in Nigerian children. Am J Phys Anthropol. 2007; 134: 443-448.

6. Lloyd S. Teething in babies: separating fact from fiction. Prof Care Mother Child. 1996; 6: 155-6.

7. Seward M. Local disturbances attributed to eruption of the human primary dentition. A survey. Br Dent J. 1971; 130: 72-77.

8. Seward M. General disturbances attributed to eruption of the human primary dentition. ASDC J. Dent. Child. 1972; 39:178-183.

9. Coreil J, Price L, Barkey N. Recognition and management of teething diarrhea among Florida pediatricians. Clin Pediatr (Phila). 1995; 34: 591-598.

10. Macknin M, Piedmonte M, Jacobs J, Skibinski C. Symptoms associated with infant teething: a prospective study. Pediatrics. 2000. 105, 747-752.

11. Peretz B, Ram D, Hermida L, Otero M. Systemic manifestations during eruption of primary teeth in infants. J Dent Child. 2003; 70: 170-173.

12. Cunha R, Pugliesi D, Garcia L, Murata S. Systemic and local teething disturbances: prevalence in a clinic for infants. J Dent Child. 2004. 71, 24-26.

13. MCintyre GT; MCintyre GM. Teething troubles? Br Dent J. 2002; 192: 251-255.

14. Olusola B, Obontu T, Michael A. Teething in infants-Knowledge and attitude of traditional birth attendants in Ibadan. Heal. 2013; 5: 1406-1411.

15. Massignan C, Cardoso M, Porporatti AL, Aydinoz S, Canto G de L, Mezzomo LA, Bolan M. Signs and symptoms of primary tooth eruption: a meta-analysis. Pediatrics. 2013. 137, 3.

16. Rocha LVA, Rocha NMO, Bullegon ALC, Perachi MI. Erupção dos dentes decíduos. Rev Gaucha Odontol. 1988; 36: 461-3.

17. Ramos-Jorge J. Sinais e sintomas associados com a erupção de dentes decíduos: estudo longitudinal [Monografia]. Belo Horizonte: Universidade Federal de Minas Gerais; 2010.

18. Carpenter JV. The relationship between teething and systemic disturbances. J Dent Child. 1978; 45: 381-4.

19. Cahill DR, Marks SC JR. Tooth eruption: evidence for the central role of the dental follicle. J Oral Pathol. 1980; 9: 189-200.

20. McCarthy PL, Sharpe MR, Spiesel SZ, Dolan TF, Forsyth BW, DeWitt TG et al. Observation scales to identify serious illness in febrile children. Pediatrics. 1982; 70: 802-809.

21. Wake M, Hesketh K, Lucas J. Teething and tooth eruption in infants: a cohort study. Pediatrics. 2000; 106: 1374-1379.

22. Ashley MP. It's only teething...a report of the myths and modern approaches to teething. Br Dent J. 2001;191; 4-8.

23. Memarpour M, Soltanimehr E, Eskandarian T. Signs and symptoms associated with primary tooth eruption: a clinical trial of nonpharmacological remedies. BMC Oral Health. 2015. 1-8.

24. Nemezio MA, De Oliveira KMH, Romualdo PC, Queiroz AM, Paula-e-Silva FWG, Silva RAB, Kuchler EC. Association between Fever and Primary Tooth Eruption: A Systematic Review and Meta-analysis. Int J Clin Pediatr Dent. 2017; 10: 293-298.

25. Seward MH. The effectiveness of a teething solution in infants. A clinical study. Brit Dent J. 1969; 127: 457-461.

26. Noor-Mohammed R, Basha S. Teething disturbances; prevalence of objective manifestations in children under age 4 months to 36 months. Med Oral Patol Oral Cir Bucal. 2012; 17: 12-15. 
27. Kakatkar G, Nagarajappa R, Bhat N, Prasad V, Sharda A, Asawa K.. Parental beliefs about children's teething in Udaipur, India: a preliminary study. Braz. Oral Res. 2012. 26, 151-157.

28. Ispas RS, Mahoney EK, Whyman RA. Teething signs and symptoms: persisting misconceptions among health professionals in New Zealand. N Z Dent J. 2013; 109: 2-5.

29. Ige OO, Olubukola PB. Teething myths among nursing mothers in a Nigerian community. Niger Med J. 2013. 54, 107-110.

30. Maia A, Prado DC, Oliveira FS De, et al. Perception of parents of children with and without disabilities about teething disturbances and practices adopted. Braz J Oral Sci. 2013;12: 76-79.

31. EL-Gilany AH, Abusaad FES. Mothers' teething beliefs and treatment practices in Mansoura, Egypt. Saudi Dent J. 2017; 8: 1-5.

32. Owais AI, Zawaideh F, Bataineh O. Challenging parents' myths regarding their children's teething. Int J Dent Hyg. 2010; 8: 28-34.

33. Wake M, Hesketh K, Allen MA. Parents beliefs about infant teething: a survey of Australian parents. J. Paediatr. Child Health. 1999; 35: 446-449.

34. Markman L. Teething: facts and fiction. Pediatr Rev. 2009; 30: 59-64.

35. Ramos-Jorge J, Pordeus IA, Ramos-Jorge ML, Paiva SM. Prospective longitudinal study of signs and symptoms associated with primary tooth eruption. Pediatrics. 2011; 128: 471-476.

36. Pierce AM, Lindskog S, Hammarstrom L. IgE in postsecretory ameloblasts suggestiong a hypersensitivity reaction at tooth eruption. J Dent Child. 1986; 53: 23-26.

37. Azevedo MS, Portela AR, Romano AR, Cenci MS. Prevalence of teething symptoms in primary teeth and associated factors: cross-sectional study in children aged 12-23 months in Pelotas, Brazil. Braz Res Paediatr Dent Integr Clin. 2015. 15, 1, 217-225.

38. Feldens CA, Faraco IM, Ottoni AB, Feldens EG, Vítolo MR. Teething symptoms in the first year of life and associated factors: a cohort study. J. clin. Pediatr. dent. 2010. 34, 3, 201-206.

39. Ramos-Jorge J, Ramos-Jorge ML, Martins-Júnior PA, Corrêa-Faria P, Pordeus IA, Paiva SM. Mothers' reports on systemic signs and symptoms associated with teething. J. Dent. Child. 2013. 80, 3, 107-110.

40. Olczak-Kowalczyk D, Turska-Szybka A, Gozdowski D, Boguszewska-Gutenbaum H, Krasuska-Slawinska E, Sobiech P, Jurczak A, Tomczyk J.. Longitudinal study of symptoms associated with teething: Prevalence and mothers' practices. Pediatria Polska. 2016. 91, 6, 533-540.

41. Bankole OO, Lawal FB. Teething: misconceptions and unhealthy practices among residents of a rural community in Nigeria. Int. q. community health educ. 2017. 37; 2: 99-106.

42. Elbur AI, Yousif MA, Albarraq AA, Abdallah MA. Parental knowledge and practices on infant teething, Taif, Saudi Arabia. BMC Res Notes. 2015; 8: 699. doi:10.1186/s13104-015-1690-y

43. Simeão MCQ, Galganny-Almeida A. Erupção dentária: estudo de suas manifestações clínicas na primeira infância segundo cuidadores e médicos pediatras. Pesqui Bras Odontopediatria Clín Integr. 2006; 6: 173180.

44. Plutzer AJ, Spencer MJNC, Keirse. How first-time mothers perceive and deal with teething symptoms: a randomized controlled trial. 1 Blackwell Publishing Ltd, Child: care, health and development. 2011. 38; 2: 292-299.

45. Cranswick N, McGillivray G. Over-the-counter medication in children: friend or foe? Australian Prescriber. 2001; 24: 149-151.

Recibido: 21/03/2019

Aceptado: 20/05/2019

Correspondencia: Rangel Bastos de Holanda Teixeira correo: rangelbaastos@hotmail.com 